\title{
Femtosecond time-resolved absorption processes in lithium niobate crystals
}

\author{
O. Beyer, D. Maxein, and K. Buse \\ Physikalisches Institut, University of Bonn, Wegelerstraße 8, D-53115 Bonn, Germany \\ B. Sturman \\ Institute of Automation and Electrometry, 630090 Novosibirsk, Russia \\ H. T. Hsieh and D. Psaltis \\ Department of Electrical Engineering, California Institute of Technology, Pasadena, California 91125
}

Received November 10, 2004

Femtosecond pump pulses are strongly attenuated in lithium niobate owing to two-photon absorption; the relevant nonlinear coefficient $\beta_{p}$ ranges from $\sim 3.5 \mathrm{~cm} / \mathrm{GW}$ for $\lambda_{p}=388 \mathrm{~nm}$ to $\sim 0.1 \mathrm{~cm} / \mathrm{GW}$ for $514 \mathrm{~nm}$. In collinear pump-probe experiments the probe transmission at the double pump wavelength $2 \lambda_{p}=776 \mathrm{~nm}$ is controlled by two different processes: A direct absorption process involving pump and probe photons $\left(\beta_{r}\right.$ $\simeq 0.9 \mathrm{~cm} / \mathrm{GW}$ ) leads to a pronounced short-duration transmission dip, whereas the probe absorption by pump-excited charge carriers results in a long-duration plateau. Coherent pump-probe interactions are of no importance. Hot-carrier relaxation occurs on the time scale of $\lesssim 0.1$ ps. (C) 2005 Optical Society of America OCIS codes: $160.3730,320.7110,190.4180$.

Lithium niobate $\left(\mathrm{LiNbO}_{3}\right)$ is a wide-gap $(\sim 4 \mathrm{eV})$ ferroelectric optical material that is of prime importance for nonlinear optical and holographic applications such as frequency conversion, optical filtering and switching, and data storage. ${ }^{1-3}$ Large $\chi^{2}$ coefficients, outstanding photorefractive properties, robustness, and availability make the material promising for realization of devices.

High intensities and correspondingly short pulses are required for efficient nonlinear optical processes and ultrafast holography. However, although $\mathrm{LiNbO}_{3}$ is well investigated in the continuous and microsecond-nanosecond ranges, ${ }^{4-7}$ almost nothing is known about its nonlinear response in the femtosecond range. It is unknown, in particular, which effects will govern the nonlinear propagation of femtosecond pulses. Free-carrier nonlinearity and absorption, Kerr nonlinearity, two-photon absorption (TPA), and photorefractive nonlinearity can compete in this range.

A crucial advantage of using femtosecond pulses as compared with picosecond and longer pulses is that the complicated processes, caused by repopulation and reexcitation of energy levels, can be separated from the direct electron excitation processes. 8 The reported results on the TPA coefficient of $\mathrm{LiNbO}_{3}$, obtained with nanosecond and subnanosecond pulses, are highly controversial ${ }^{10}$; they have a range of more than 1 order of magnitude at $\sim 530 \mathrm{~nm}$. Thus for practical purposes and also for physics it is highly important to explore femtosecond time-resolved absorption processes in $\mathrm{LiNbO}_{3}$, which is the topic of this work.

The conditions of our collinear pump-probe experiments are as follows: A single axially symmetric pump pulse of wavelength $\lambda_{p}=388-776 \mathrm{~nm}$, extracted from a Ti:sapphire femtosecond laser (CPA-
2010, Clark-MXR plus an optical parametric amplifier system), is incident normally onto the $Y Z$ face of a $\mathrm{LiNbO}_{3}$ sample; the polar $\mathbf{c}$ axis is parallel to the $Z$ axis. The pulse width and beam diameter (at the half-intensity) are $\sim 0.24 \mathrm{ps}$ and $\sim 0.6 \mathrm{~mm}$, respectively, for $388 \mathrm{~nm}$. Peak intensity $I_{p}^{0}$ ranges from $\sim 1$ to $\sim 276 \mathrm{GW} / \mathrm{cm}^{2}$. The maximum pump fluence is approximately $59 \mathrm{~mJ} / \mathrm{cm}^{2}$. The input polarization vector of the pump pulse is either parallel or perpendicular to the c axis. Thickness $d$ for three investigated (undoped and congruently melting) samples is $1,0.5$, and $0.07 \mathrm{~mm}$. In the wavelength range used, the linear absorption is negligibly small. In addition to the pump transmission, the transmission coefficient for a weak probe pulse $\left(\lambda_{r}=2 \lambda_{p}=776 \mathrm{~nm}\right)$ is measured as a function of its time delay $\Delta t$. The probe pulse width and diameter are not essentially different from those of the pump.

Before presenting our experimental data, we consider the pump transmission coefficient in the presence of TPA. Pump intensity $I_{p}$ obeys the nonlinear wave equation

$$
\left(\frac{\partial}{\partial x}+\frac{1}{v_{p}} \frac{\partial}{\partial t}\right) I_{p}=-\beta_{p} I_{p}^{2},
$$

where $x$ is the propagation coordinate, $\beta_{p}$ is the TPA coefficient for the pump pulse, and $v_{p}=c / n_{p}$ is the pulse velocity with $n_{p}$ being the relevant refractive index. Equation (1) follows from Maxwell's equations under the assumption of negligible diffraction broadening and frequency dispersion, which is well justified for the experimental conditions. Equation (1) has to be solved with a boundary condition at the input $x=0$; here we use a Gaussian profile $I_{p}(x=0)$ $=I_{p}^{0} \exp \left[-\left(t / t_{p}\right)^{2}-\left(r / r_{p}\right)^{2}\right]$, where $I_{p}^{0}$ is the input peak 


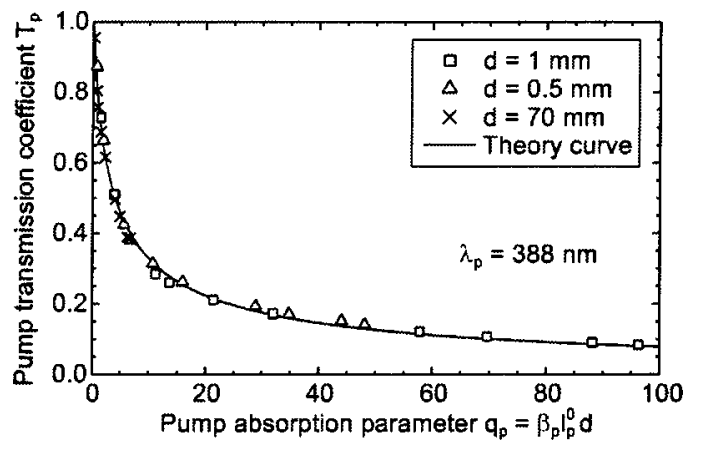

Fig. 1. Dependence of normalized transmission coefficient $T_{p}$ on the product $\beta_{p} I_{p}^{0} d$. The squares, triangles, and crosses correspond to the data obtained for $d=1,0.5$, and $0.07 \mathrm{~mm}$.

intensity; $r$ is the radial coordinate; and $r_{p}$ and $t_{p}$ characterize the pump beam radius and the pulse half-width, respectively. The solution of Eq. (1) meeting this boundary condition is given by

$$
\frac{I_{p}^{0}}{I_{p}}=\beta_{p} I_{p}^{0} x+\exp \left[\frac{\left(t-x v_{p}^{-1}\right)^{2}}{t_{p}^{2}}+\frac{r^{2}}{r_{p}^{2}}\right] .
$$

It describes the propagation of the pump pulse with velocity $v_{p}$, the decrease in the peak intensity, and flattening of the top of the pulse (in the transverse directions and in time) owing to the preferential absorption at the beam center.

To find the normalized transmission coefficient for the pump, $T_{p}$, we integrate output intensity $I_{p}(d, r, t)$ over time and the transverse coordinates and divide the quantity obtained by its value at $d=0$. The final result is

$$
T_{p}=\frac{2}{q_{p} \sqrt{\pi}} \int_{0}^{\infty} \ln \left[1+q_{p} \exp \left(-\xi^{2}\right)\right] \mathrm{d} \xi,
$$

with $q_{p}=\beta_{p} I_{p}^{0} d$ (see also Ref. 9). The transmission thus depends only on the product $\beta_{p} I_{p}^{0} d$. The solid curve in Fig. 1 shows calculated dependence $T_{p}\left(q_{p}\right)$. This dependence is rather specific. After a rapid initial fall, the further decrease of $T_{p}$ occurs slowly; this is indeed due to the relatively weak energy absorption on the pulse wings.

The squares, triangles, and crosses in Fig. 1 represent experimental data for the samples with $d=1$, 0.5 , and $0.07 \mathrm{~mm}$, respectively. The only variable parameter to fit the theoretical curve, Eq. (3), to all the experimental data is the TPA coefficient $\beta_{p}$. Excellent agreement between theory and experiment within the large intensity range is evident. This proves unambiguously that TPA is the reason for the pump attenuation. The estimated value of the TPA coefficient at $388 \mathrm{~nm}$ is $\beta_{p} \sim 3.5 \mathrm{~cm} / \mathrm{GW}$. The maximum value of the product $\beta_{p} I_{p}^{0} d$ is approximately 96,48 , and 7 for the samples with $d=1,0.5$, and $0.07 \mathrm{~mm}$, respectively; the pump pulse is subjected to a strong nonlinear absorption even in relatively thin samples.

In a similar manner, the values of $\beta_{p}$ have been deduced for different pump wavelengths. The corre- sponding data are presented by circles in Fig. 2. One sees from Fig. 2 that the TPA coefficient decreases strongly as the wavelength increases. For $\lambda_{p}$ $\gtrsim 600 \mathrm{~nm}$ the energy of two photons is insufficient for band-band transitions and $\beta_{p}$ approaches zero.

Note that the previous TPA measurements for $\mathrm{LiNbO}_{3}$ crystals were performed with nanosecond and subnanosecond pulses. The corresponding data for $\beta_{p}$ are highly controversial, ${ }^{10}$ probably because of a strong influence of secondary excitation processes typical for this material. ${ }^{5,6}$ The energy of our femtosecond pulses is insufficient for such processes.

Now we turn to our experimental data on the probe transmission. Figure 3 shows the dependence of probe transmission coefficient $T_{r}$ on time delay $\Delta t$ between the pump pulse and the probe pulse for $I_{p}^{0}$ $\sim 107 \mathrm{GW} / \mathrm{cm}^{2}, d=0.07 \mathrm{~mm}$, and four different combinations of beam polarizations. It includes a great deal of information about the light-matter interactions. Dependence $T_{r}(\Delta t)$ is characterized by a narrow dip (its width is comparable with the pulse width) followed by a quasi-permanent section (plateau). The plateau value of $T_{r}$ remains unchanged at least on the scale of $10^{-11} \mathrm{~s}$. The dip amplitude is largest in the cases of parallel polarization of the pump and probe $(Z Z$ and $Y Y$ ); the influence of the orientation of the polarization plane is fairly weak. In

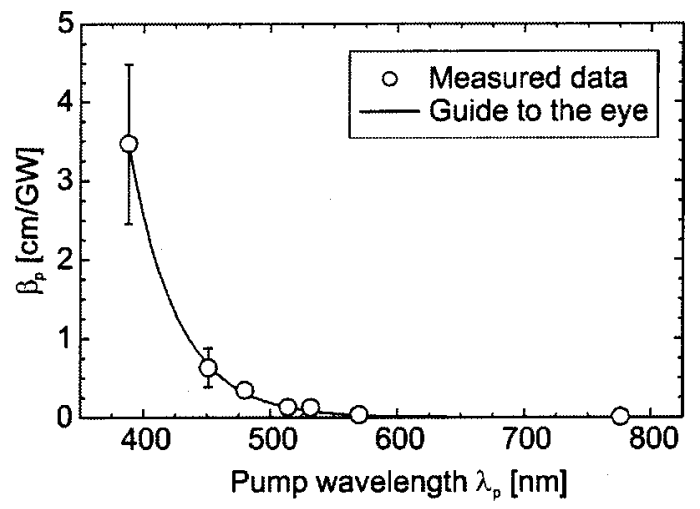

Fig. 2. Dependence of quadratic absorption coefficient $\beta_{p}$ on pump wavelength $\lambda_{p}$.

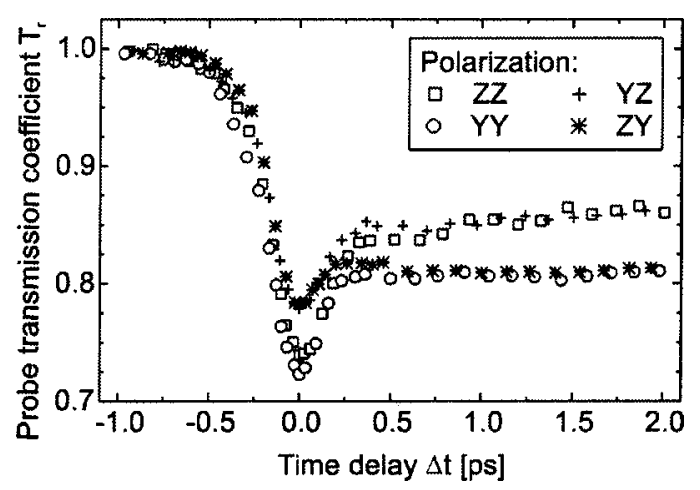

Fig. 3. Normalized transmission coefficient $T_{r}$ for the probe pulse versus time delay $\Delta t$; first and second symbols in the inset specify the orientation of the polarization vector of the pump and the probe, respectively. 
the cases of orthogonal polarizations of the pump and probe ( $Y Z$ and $Z Y$ ) the dip is less pronounced. The plateau value of $T_{r}$ is determined by the probe polarization; the absorption effect is slightly larger for a $Y$-polarized probe beam.

The plateau section in $T_{r}(\Delta t)$ (Fig. 3) is attributed to the probe absorption by the carriers (electrons and/or holes) excited through two-photon pump absorption. It is known from experiments with $\mathrm{LiNbO}_{3}$ crystals and nanosecond pulses that excitation of charge carriers causes light absorption that persists for some microseconds. ${ }^{6,11}$ Some polarization dependence of the plateau value is explained by the crystal anisotropy.

The dip in $T_{r}(\Delta t)$ contrasts with the effect of decreasing probe absorption owing to coherent parametric pump-probe interactions that are known for a number of semiconductors (see Ref. 12 and references therein). The increase in the probe absorption in the presence of the pump pulse is of different origin. We attribute it to two-photon transitions involving both pump and probe photons; their total energy $(\sim 4.8 \mathrm{eV})$ exceeds the width of the forbidden gap of $\mathrm{LiNbO}_{3}$ crystals. Identical polarizations of the pump and probe facilitate this direct process.

The dependences of the dip amplitude and the plateau absorption $1-T_{r}^{\text {plat }}$ on the pump intensity are different. Although the dip amplitude tends to saturate with increasing $q_{p}=\beta_{p} I_{p}^{0} d$ the plateau absorption shows a superlinear behavior in the range $q_{p}=1-8$. This is explained by different orders (in $I_{p}^{0}$ ) of the relevant nonlinear processes. The pump-induced TPA coefficient for the probe pulse and the absorption cross section of the pump-excited carriers at $\lambda_{r}$ estimated from our experimental data are $\beta_{r}$ $\approx 0.9 \mathrm{~cm} / \mathrm{GW}$ and $\sigma_{r} \approx 0.8 \times 10^{-17} \mathrm{~cm}^{-2}$, respectively. These quantities do not show strong polarization sensitivity.

The data on dependence $T_{r}(\Delta t)$ show no indication of the relaxation of hot charge carriers excited during the two-photon processes: The dip is almost symmetric and no features with a characteristic time different from the pulse duration time are present. The relaxation time of nonthermalized carriers in solids is expected to be in the range $0.1-10 \mathrm{ps}^{8}{ }^{8}$ Most probably, the 0.1-ps boundary of this interval is relevant to $\mathrm{LiNbO}_{3}$ crystals.

With the Kerr coefficients expected for lithium niobate ${ }^{13}$ the light-induced index changes cannot affect the pump-probe transmission. The situation should be different in the case of nonlinear wave mix- ing and grating recording. Since the TPA coefficients of $\mathrm{LiNbO}_{3}$ are high, both absorption and index gratings have to be involved in Bragg-diffraction processes.

In conclusion, we have shown that TPA processes strongly affect the propagation of subpicosecond light pulses in lithium niobate crystals within the bluegreen spectral range. The measured TPA coefficient is a strongly decreasing function of the wavelength, and overlap of pump and probe pulses leads to decreasing probe transmission owing to combined TPA transitions. This phenomenon is opposite to the effect of increasing probe transmission owing to coherent pump-probe interactions known in semiconductors. Excitation of free carriers results in long-lasting light absorption, and hot-carrier relaxation cannot be resolved on the scale of $10^{-13} \mathrm{~s}$. Competition of TPA and Kerr gratings is expected to be important for femtosecond holography phenomena.

Financial support from the Deutsche Telekom AG, the Deutsche Forschungsgemeinschaft (award BU 913/13-1), and the National Science Foundation (award EEC-9402726 and award INT-0233988) is gratefully acknowledged. O. Beyer's e-mail address is beyer@physik.uni-bonn.de.

\section{References}

1. M. M. Fejer, G. A. Magel, D. H. Jundt, and R. L. Byer, IEEE J. Quantum Electron. 28, 2631 (1992).

2. P. Boffi, D. Piccinin, and M. C. Ubaldi, Infrared Holography for Optical Communications (SpringerVerlag, Berlin, 2003).

3. H. J. Coufal, D. Psaltis, and G. T. Sincerbox, eds., Holographic Data Storage (Springer, New York, 2000).

4. L. Solymar, D. J. Webb, and A. Grunnet-Jepsen, The Physics and Applications of Photorefractive Materials (Clarendon, Oxford, 1996).

5. K. Buse, Appl. Phys. B 64, 391 (1997).

6. F. Jermann and J. Otten, J. Opt. Soc. Am. B 10, 2085 (1993).

7. C. T. Chen, D. M. Kim, and D. von der Linde, Appl. Phys. Lett. 34, 321 (1979).

8. A. Othonos, J. Appl. Phys. 83, 1789 (1998).

9. A. Dragonmir, J. G. McInerney, and D. N. Nikogosyan, Appl. Opt. 41, 4365 (2002).

10. D. N. Nikogosyan, Properties of Optical and LaserRelated Materials (Wiley, New York, 1997), p. 98.

11. D. Berben, K. Buse, S. Wevering, P. Herth, M. Imlau, and Th. Woike, J. Appl. Phys. 87, 1034 (2000).

12. H. J. Eichler, P. Günter, and D. W. Pohl, Laser-Induced Dynamic Gratings (Springer-Verlag, New York, 1986).

13. R. DeSalvo, A. A. Said, D. J. Hagan, E. W. Van Stryland, and M. Sheik-Bahae, IEEE J. Quantum Electron. 32, 1324 (1996). 